\title{
Syndrome in question: antisynthetase syndrome (anti-PL-7) ${ }^{*}$
}

\author{
Ana Cláudia Cavalcante Esposito ${ }^{1}$ \\ Hélio Amante Miot ${ }^{1}$
}

\author{
Tatiana Cristina Gige ${ }^{1}$
}

\section{CASE REPORT}

A woman patient, 41 years of age, presented a dry cough and dyspnea upon moderate exertion, which has become progressively worse over the past four years. Six months ago, the patient presented proximal muscle weakness and wrist arthralgia. She sought out the Dermatology department due to a peeling of the edges of the fingers, without pruritus; periungual erythema; changes in nails and fingers (Figure 1). Physical examination identified a crackling sound in the lungs. On the hands, one could observe Raynaud phenomenon, peeling, and marginal hyperqueratosis ("mechanic's hands"); longitundinal hypercurvature of the nail plate; paronychia; reduction in the volume of finger tissue (digital pulp), with no ulcer- ations, sclerodactyly or punctiform scars. Capillaroscopy showed folding, ischemic areas, and dilated flaps, as well as the absence of heliotrope. Laboratory exams included FAN 1:160 (speckled) and positive anti-PL-7. Other antibodies included antisynthetase, antinuclear, anti-Scl-70, ANCA, and latex proved to be normal. Biochemical exams - VHS 122mm/h; Creatine phosphokinase 2759U/1 (reference<135U/1). Electroneuromyography showed a myopathic pattern. Computed tomography of the thorax showed interstitial lung disease (ILD) with ground-glass opacity (Figure 2); spirometry disclosed a restrictive pattern, with no response to the bronchodilator, and the esophagogram was normal.
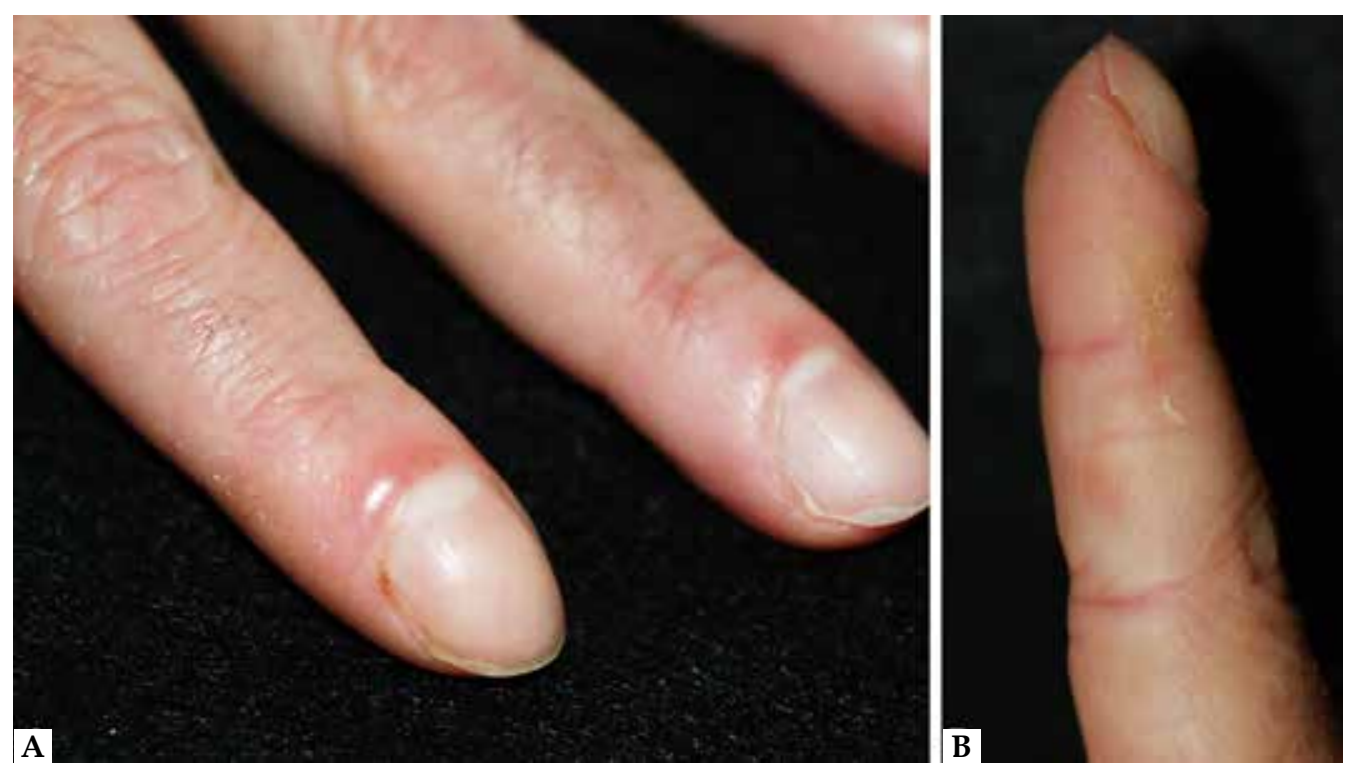

Figure 1:

A. Periungual erythema, longitudinal hypercurvature of the ungual lamina and marginal hyperkeratosis of the first finger. B. Detail of the nail hypercurvature (digital clubbing), retraction of digital pulp, and marginal peeling of the second finger.(mechanic's hand)

\section{Received on 08.02.2015}

Approved by the Advisory Board and accepted for publication on 22.04.2015

Work conducted in the Department of Dermatology and Radiology of Universidade Estadual Paulista "Júlio de Mesquita Filho" (Unesp) - Botucatu Campus, Botucatu, SP, Brazil.

Financial Support: None.

Conflict of Interest: None

1 Universidade Estadual Paulista “Júlio de Mesquita Filho” (Unesp), Botucatu, SP, Brazil.

@2016 by Anais Brasileiros de Dermatologia 


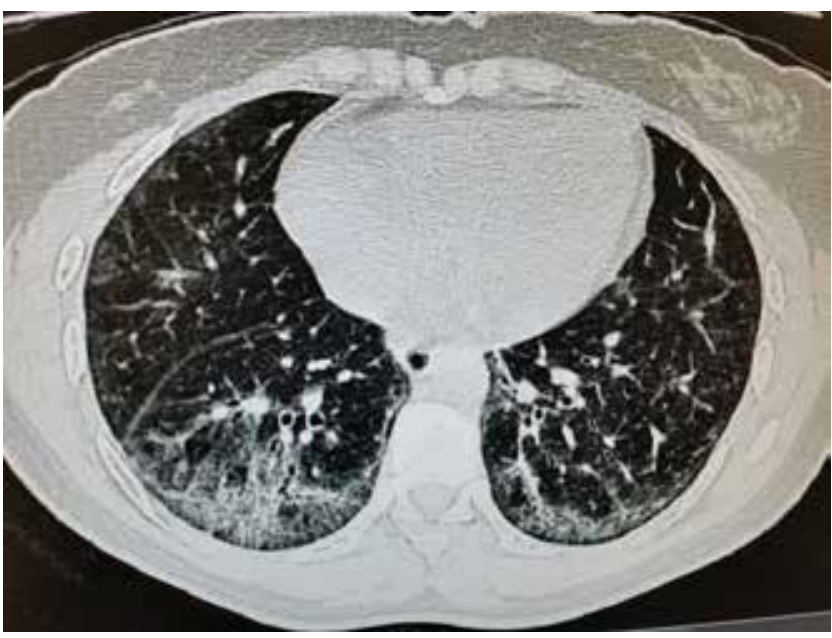

Figure 2: Computed tomography (high resolution) of the thorax. Fibrosing interstitial pneumonia with a reticular pattern with areas of ground-glass alveolar opacity and thickness of the septa (inter and intralobuar) with traction bronchiectasis, predominantly in the lower basal regions
The autoantibodies involved in the disease (in order of frequency) and the amino acids that are targets of RNAt-synthetase are: anti-Jo1 (histidine), anti-PL-12 (alanine), anti-PL-7 (threonine), anti-OJ (isoleucine), anti-KS (asparagine), anti-EJ (glycine), and anti-ZO (phenylalanine). The phenotype of the disease varies according to the expressed autoantibody. ${ }^{7}$ Myopathy occurs more often in patients with anti-Jo-1 or anti-PL-7; anti-Jo-1 is related to the more extreme cases of arthritis and "mechanic's hand", while anti-PL-7 is associated with the presence of heliotrope; anti-PL-12 with the higher rates of Raynaud phenomenon; and anti-PL-12, anti-KS, and anti-OJ with cases of isolated ILD. ${ }^{8}$

To date, no randomized studies have evaluated the therapeutic effectiveness of ASS. ${ }^{7}$ The treatment of choice is prednisone at an initial dose of $1 \mathrm{mg} / \mathrm{Kg} /$ day. Other possibilities described include: tacrolimus, mycophenolate mofetil, immunoglobin, azathioprine, methotrexate, cyclophosphamide, and cyclosporine. ${ }^{7}$ Rituximab was reported to be efficient in the treatment of refractory myositis, but did not influence the evolution of ILD or the loss of

CHART 1: Criteria proposed for the diagnosis of antisynthetase syndrome ${ }^{3}$

Diagnosis: Presence of one antisynthetase antibody plus two major criteria or one higher criterion and two minor criteria.

\section{Major criteria:}

1. Interstitial lung disease (not explained by environmental, occupational, medication exposure, and not related to any other base disease)

2. Polymyositis or dermatomyositis according to criteria set forth by Bohan \& Peter

\section{Minor criteria:}

1. Arthritis

2. Raynaud phenomenon

3. Mechanic's hand

\section{DISCUSSION}

Antisynthetase Syndrome (ASS), described by Marguerie (1990), represents a rare group of autoimmune diseases that mainly affect women (2:1) between 30 and 50 years of age and present autoantibodies geared toward the Amino Acyl-tRNA Synthetases enzymatic complex, which catalyzes the connection of an amino acid to the RNAt protein synthesis. ${ }^{1-5}$

The diagnostic criteria of ASS are available in chart $1 .^{3}$ The most common clinical manifestations of ASS include: inflammatory myositis (>90\%), Interstitial Lung Disease (ILD) $(60 \%)$, non-erosive arthritis (50\%), Raynaud phenomenon (40\%), "mechanic's hand" $(30 \%)$, and fever $(20 \%) .{ }^{6}$ The main differential diagnoses include progressive systemic sclerosis, dermatomyositis/polymyositis, and overlap syndromes. pulmonary function. ${ }^{1,9}$

The prognosis of ASS is quite limited when compared to other inflammatory myopathies without antisynthetase antibodies, which results mainly from greater ILD resistance to corticotherapy, evolving into the need for a lung transplant. ${ }^{1,3,10}$

Our patient was submitted to oral corticotherapy associated with pulse therapy using cyclophosphamide and, later, with mycophenolate mofetil and rituximab, without, to date, fully controlling the lung disease.

Dermatologists must pay attention to myopathic and/or lung conditions that present the Raynaud phenomenon, nail dystrophy, repeated periungual/paronychia erythema, atrophy of the digital pulp, and "mechanic's hand" peeling, in an attempt to collaborate with an early diagnosis of the disease with a limited prognosis $\square$ 
Abstract: Antisynthetase syndrome is a rare autoimmune disease characterized by interstitial lung disease and/or inflammatory myositis, with positive antisynthetase antibodies (anti-Jo-1, anti-PL-7, anti-PL-12, ZO, OJ, anti-KE or KS). Other symptoms described include: non-erosive arthritis, fever, Raynaud's phenomenon, and "mechanic's hands." The first therapeutic option is corticotherapy, followed by other immunosuppressants. The prognosis of the disease is quite limited when compared to other inflammatory myopathies with negative antisynthetase antibodies.

Keywords: Amino Acyl-tRNA Synthetases; Interstitial Lung Disease; Myositis

\section{REFERENCES}

1. Love LA, Leff RL, Fraser DD, Targoff IN, Dalakas M, Plotz PH, et al. A new approach to the classification of idiopathic inflammatory myopathy: myositisspecific autoantibodies define useful homogeneous patient groups. Medicine (Baltimore). 1991;70:360-74.

2. Marguerie C, Bunn CC, Beynon HL, Bernstein RM, Hughes JM, So AK, et al. Polymyositis, pulmonary fibrosis and autoantibodies to aminoacyl-tRNA synthetase enzymes. Q J Med. 1990;77:1019-38.

3. Solomon J, Swigris JJ, Brow KK. Doença pulmonar intersticial relacionada a miosite e a síndrome antissintetase. J Bras Pneumol. 2011;37:100-109.

4. Ortigosa LCM, Reis VMS. Dermatomiosite. An Bras Dermatol 2008;83:247-59.

5. Fischer A, Swigris JJ, du Bois RM, Lynch DA, Downey GP, Cosgrove GP, et al. Anti-synthetase syndrome in ANA and anti-Jo-1 negative patients presenting with idiopathic interstitial pneumonia. Respir Med. 2009;103:1719-24.

6. Foris V, Kovacs G, Matucci-Cerinic M, Olschewski H. PL-7 positive antisynthetase syndrome and pulmonary hypertension. J Rheumatol. 2013;40:1777-9.

7. Hallowell RW, Danoff SK. Interstitial lung disease associated with the idiopathic inflammatory myopathies and the antisynthetase syndrome: recent advances. Curr Opin Rheumatol. 2014;26:684-9.

8. Dugar M, Cox S, Limaye V, Blumbergs P, Roberts-Thomson PJ. Clinical heterogeneity and prognostic features of South Australian patients with antisynthetase autoantibodies. Intern Med J. 2011;41:674-9.

9. Nalotto L, laccarino L, Zen M, Gatto M, Borella E, Domenighetti M, et al. Rituximab in refractory idiopathic inflammatory myopathies and antisynthetase syndrome: personal experience and review of the literature. Immunol Res. 2013;56:362-70.

10. Behr J, Ryu JH. Pulmonary hypertension in interstitial lung disease. Eur Respir J. 2008;31:1357-67.

\author{
MAILING ADDRESS: \\ Hélio Amante Miot \\ Departamento de Dermatologia e Radioterapia \\ Faculdade de Medicina de Botucatu \\ Unesp \\ 18618-000 Botucatu, SP \\ E-mail: heliomiot@fmb.unesp.br
}

How to cite this article: Esposito ACC, Gige TC, Miot HA. Syndrome in question: antisynthetase syndrome (antiPL-7). An Bras Dermatol. 2016; 91(5):683-5. 